\title{
LIII. On the passage of electric wave-trains through layers of electrolyte
}

\section{G. Udny Yule}

To cite this article: G. Udny Yule (1893) LIII. On the passage of electric wave-trains through layers of electrolyte, Philosophical Magazine Series 5, 36:223, 531-544, DOI: 10.1080/14786449308620509

To link to this article: http://dx.doi.org/10.1080/14786449308620509

曲 Published online: 08 May 2009.

Submit your article to this journal $[\pi$

Џ Article views: 2

Q View related articles $\asymp$ 
molecules that can be explained by representing the molecules as smooth spheres, but there are others which cannot be so explained, the best known of the latter being the specific heat. It is well to see how far a simplifying hypothesis such as that of smooth spherical molecules can lead us; but it is also well to recognize when the hypothesis has got to the end of its tether. Van der Waals's theoretical equation agrees closely with experiment for the element gases only through an accidental compensation in the effects of two neglected causes, namely, the effect of molecular force on the number of collisions of molecules and the effect of a difference between the forces called into play in the collision of molecules and of smooth spheres. It must also be remembered that the empirical equation given for compound gases in my paper on the "Laws of Molecular Force" is quite different in form from that for elements, and that a theoretical explanation of it must involve considerations beyond the range of the spherical molecule. Indeed it appears to me that a combined and collated study of specific heat, characteristic equation, and thermal conductivity of gases might now be expected to yield some of that knowledge of the internal dynamics of molecules which is absolutely necessary for the advancement of the kinetic theory in the most interesting directions.

Melbourne, June 1893.

LIII. On the Passage of Electric Wave-trains through Layers of Electrolyte. By G. UDNY YULE*.

Introduction.

THE attempt to compare the resistances of electrolytes with 1 rapidly alternating currents, by utilizing for that purpose electric radiation, was first made by Prof. J. J. Thomson in $1888 \uparrow$. The method he used was as follows:-Between a circular oscillator and a resonator was placed a large shallow dish, into which an electrolyte was poured, forming an absorbent layer which greatly weakened the resonator-sparks and finally extinguished them. So long as the layer be thin, the thickness of liquid necessary to just extinguish the sparks is inversely proportional to its conductivity. In this way the conductivities of several different solutions were compared, and the ratios found were approximately those of the conduc-

- Communicated by the Author. A preliminary note was published, Proc. Roy. Soc. liv. p. 96, May 1893.

$\dagger$ Proc. Roy. Soc. xlv. p. 269 (1889). 
tivities determined by ordinary methods. No absolute determinations, however, could be made, and the measurements were naturally somewhat rough.

Since it would be important to make certain of even a slight variation in the resistance with increasing rapidity of oscillation, I decided last winter to take up these experiments again, with such alterations as might suggest themselves to render measurements more exact. After several unsuccessful attenipts to directly improve the procedure used by Prof. J. J. Thomson, the following method was adopted:-The waves were propagated along (or between) a pair of long wires instead of being allowed to scatter in all directions through space; a certain length of these wires was then immersed in an electrolyte, and the ratio determined in which the wavetrains were weakened by their passage tbrough this absorbent layer. Measurements could be fairly accurately made with an electrometer. It would have been easy from a series of observations to calculate the condnctivity of the electrolyte used, if the change in the transmitted intensity were only a consequence of absorption. But the matter proved not so simple. As the thickness of the absorbent layer increased, the transmitted intensity, so far from decreasing logarithmically, did not continuously decrease at all, but decreased and increased periodically. The effect was obviously analogous to the interference-phenomena of thin plates by transmitted light: independently of any slight absorption a layer a quarter wave-length thick transmitted a minimum, a half wave-length thick a maximum, and so on. This disturbance made the matter too complex to permit of an exact determination of the conductivity, but its intrinsic interest was quite sufficient to warrant further investigation, and I give in the following pages the detailed results of my experiments.

\section{Apparatus.}

Fig. 1 represents the actual apparatus diagrammatically. The primary and secondary conductors were given the form

Fig. 1.

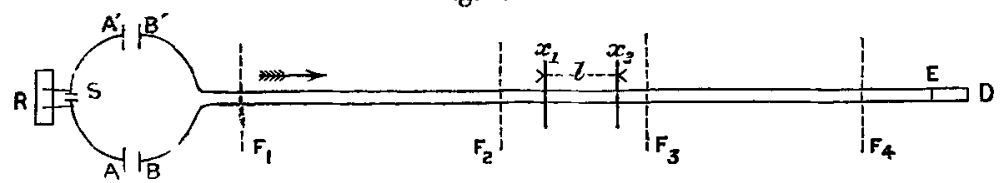

and dimensions previously used by Bjerknes*. The primary oscillator consisted of two circular zinc plates, $\mathrm{A} \mathrm{A}^{\prime}$, connected

* Wied. Ann. xliv. p. 513 (1891). 
to the spark-gap, S, by wires, A S, A'S, and was worked by a Ruhmkorff coil, R. Opposite the plates A $A^{\prime}$ stood similar plates B B', from which a pair of long wires B F D were led off, stretched parallel to each other. These wires serve simply as guides for the radiation, which travels straight up the space between them and through the electrolyte $x_{1}, x_{2}$.

The principal dimensions of the oscillator \&c. were as follows :-

Diameter of plates $\mathrm{A} \mathrm{A}^{\prime}, \mathrm{B} \mathrm{B}^{\prime}$. . . 40 centim. Distance A B, $\mathrm{A}^{\prime} \mathrm{B}^{\prime}$. . . . . . 30 ", Length of wire $\mathrm{AS} \mathrm{A}$ (diam. 2 millim.) 200 ", Wave-length $\lambda$. . . . . . 900 ,

The wires BFD (about 1 millim. diam.) were spanned 6 centim. apart. If these wires be made too short, a wavetrain emitted from $B B^{\prime}$ may be reflected at the electrolyte $x_{1}$ or the bridge $\mathrm{D}$ and arrive back at $\mathrm{B}$ before the primary has finished oscillating. If this occur, alterations in the state of the secondary may react on the primary somewhat as in an alternate-current transformer, and inconvenient interference takes place. But if $\mathrm{B} x_{1}$ be made longer than half the effective length of the wave-train, the reflected waves will not reach $B$ until the primary has practically finished oscillating, and the latter can then know notbing of any alterations in the sccondary at or beyond $x_{1}$. Such a reaction of the secondary on the primary was noticed to a very serious extent by Herr von Geitler* with a Blondlot oscillator $\dagger$.

In my apparatus the wires were led out at $F_{1}$ in a circuit of about 50 metres circumference round a garden next the laboratory. They re-entered the room at $\mathrm{F}_{2}$, and were then run vertically through the vessel containing the electrolyte $x_{1}, x_{2}$. A second length of 50 metres, $\mathrm{F}_{3} \mathrm{~F}_{4}$, round the garden, completed the circuit, the wires re-entering the room at $\mathrm{F}_{4}$ and being bridged at, $\mathrm{D}$. The electrometer was connected at $\mathrm{E}$ a quarter wave-length $(2 \cdot 25$ metres $)$ from the end of the wires, at the crest of the standing waves formed by interference of the direct and reflected trains. If we accept, at least as an approximation, the value of the primary damping determined by Bjerknes (loc. eit.) for his apparatus, these dimensions should suffice to exclude any sensible reaction.

The electrometer was the one employed by Bjerknes for his researches in the same laboratory. It is a simpls quadrant-electrometer with only one pair of quadrants and an

* Doctor-Dissertation : Bonn, Jan. 1893, p. 22.

$\dagger$ Comptes Rendus, cxiv. p. 283 (1892). 
aluminium needle of the usual shape suspended by a quartz fibre. One quadrant is connected to each wire. Tihe needle not being charged, throws are always in the same direction, and are directly proportional to the time-integral of the energy, or to the intensity of the radiation. First throws, not steady deflexions, are read.

Various glass jars were used for holding the electrolyte. The wires were run vertically through holes in the bottom of the jar, strained straight, and cemented in.

\section{Experiments.}

Electrometer-readings were taken in pairs alternately with the dish empty and with, say, 1 centim. of liquid in it, then alternately with the dish empty and with 2 centim. of liquid, and so on, a good many readings being taken at each point to get a good average and eliminate spark-variations. By this means one gets a curve showing the variation in the transmitted intensity as the thickness of the layer is increased, the intensity transmitted when the thickness is zero being always taken as unity.

Fig. 2.

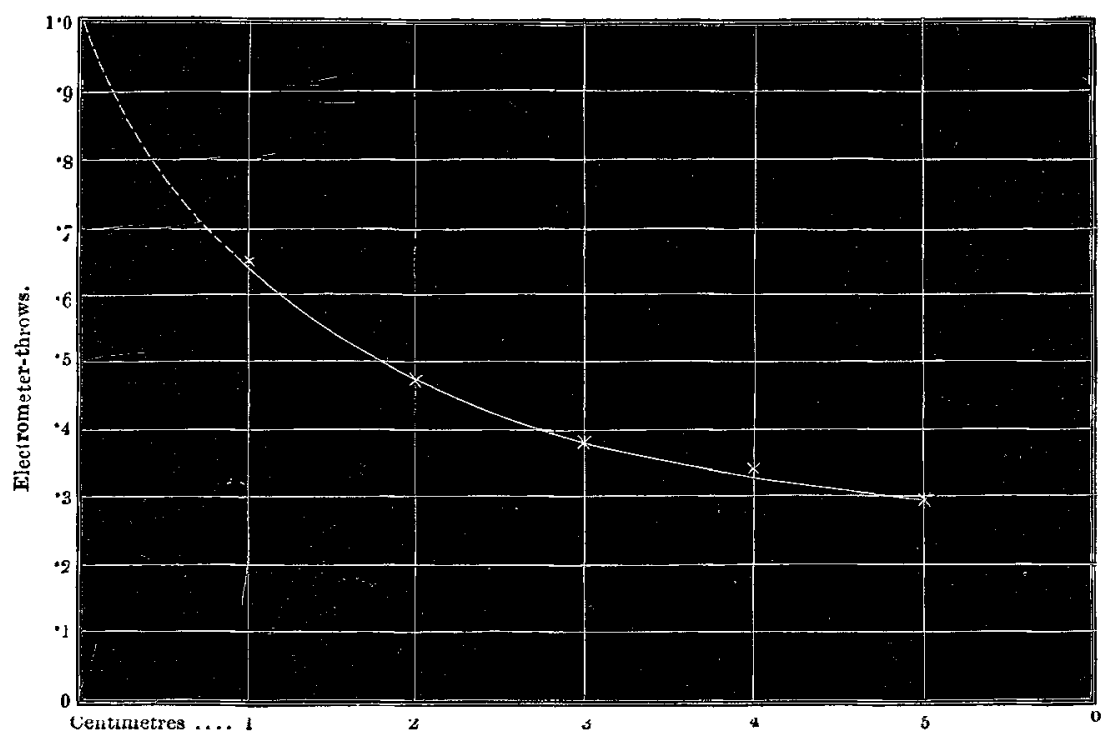

Transmission-curve for a thin layer $\mathrm{CuSO}_{4}$ solution. 
The first trials of the apparatus were made with dilute solutions of copper sulphate, some five or six centimetres of solution being the greatest depth used, as the dish was only a shallow one. One of the first curves obtained is given in fig. 2, and may serve as a type of two or three similar ones. The curve is by no means logarithmic, and if the conductivity of the solution be calculated from a pair of points on it arbitrarily chosen near the beginning and end it comes out very high.

It appeared possible that this irregularity might be due to the existence of interference-effects analogous to Newton's rings or the phenomena of thin plates by transmitted light, especially in view of a somewhat similar effect observed just previously by Mr. Barton in the same laboratory *; but I felt doubtful at first whether it might not be ascribed to some other disturbance - to the fact that a part of the radiation passes round outside the jar instead of through the electrolyte, or to mutual induction between the two halves of the long wire circuit in the garden. The second disturbance proved, however, to be nonexistent, and the first quite inadequate to explain such a distortion of the curve.

To see if the effact were really due to interference phenomena, it was necessary to determine a transmission-curve for a great length of liquid, and consequently to avoid absorption as far as possible. Distilled water presented itself naturaily as the best liquid for the purpose.

A tall glass cylinder, about 114 centim. high and 12 centim. internal diameter, was used as the containing vessel, the wires being strained straight down the middle of it, and passing through holes drilled in the bottom.

In order to avoid having to remove all the water between every pair of readings, the method of observation was slightly altered : each point was compared with one previously deter. mined, instead of directly with the zero. As before, ten or tweilve readings were taken at every point to cover variations in the effectiveness of the primary sparks. As a specimen of the usual variations, I give two series of observations in Table I. The first was for the determination of the ratio between the intensities transmitted at 40 and at 55 centim., the second for the determination of the same ratio at 60 and 80 centim.

* Proc. Roy. Soc. liv. p. 85 (1893). 


\section{TABLE I.}

\begin{tabular}{|c|c||c|c|}
\hline 40 centim. & 55 centim. & 60 centim. & 80 centim. \\
\hline $4 \cdot 6$ & $11 \cdot 4$ & $6 \cdot 8$ & $3 \cdot 0$ \\
$4 \cdot 9$ & $11 \cdot 4$ & $6 \cdot 4$ & $3 \cdot 0$ \\
$5 \cdot 0$ & $11 \cdot 0$ & $6 \cdot 5$ & $2 \cdot 8$ \\
$4 \cdot 2$ & $11 \cdot 9$ & $6 \cdot 1$ & $2 \cdot 7$ \\
$4 \cdot 3$ & $11 \cdot 5$ & $6 \cdot 1$ & $2 \cdot 3$ \\
$3 \cdot 9$ & $11 \cdot 2$ & $6 \cdot 1$ & $2 \cdot 5$ \\
$4 \cdot 0$ & $11 \cdot 6$ & $5 \cdot 9$ & $2 \cdot 7$ \\
$4 \cdot 3$ & $11 \cdot 4$ & $5 \cdot 7$ & $2 \cdot 5$ \\
$4 \cdot 6$ & $10 \cdot 4$ & $5 \cdot 8$ & $2 \cdot 0$ \\
$4 \cdot 4$ & $11 \cdot 2$ & $6 \cdot 6$ & $2 \cdot 1$ \\
$4 \cdot 5$ & $10 \cdot 4$ & $5 \cdot 8$ & $2 \cdot 7$ \\
$4 \cdot 6$ & $10 \cdot 0$ & $6 \cdot 8$ & $2 \cdot 7$ \\
\hline
\end{tabular}

The readings for each thickness are grouped separately, but it will be understood that they were taken in pairs alternately as described above.

Fig. 3.

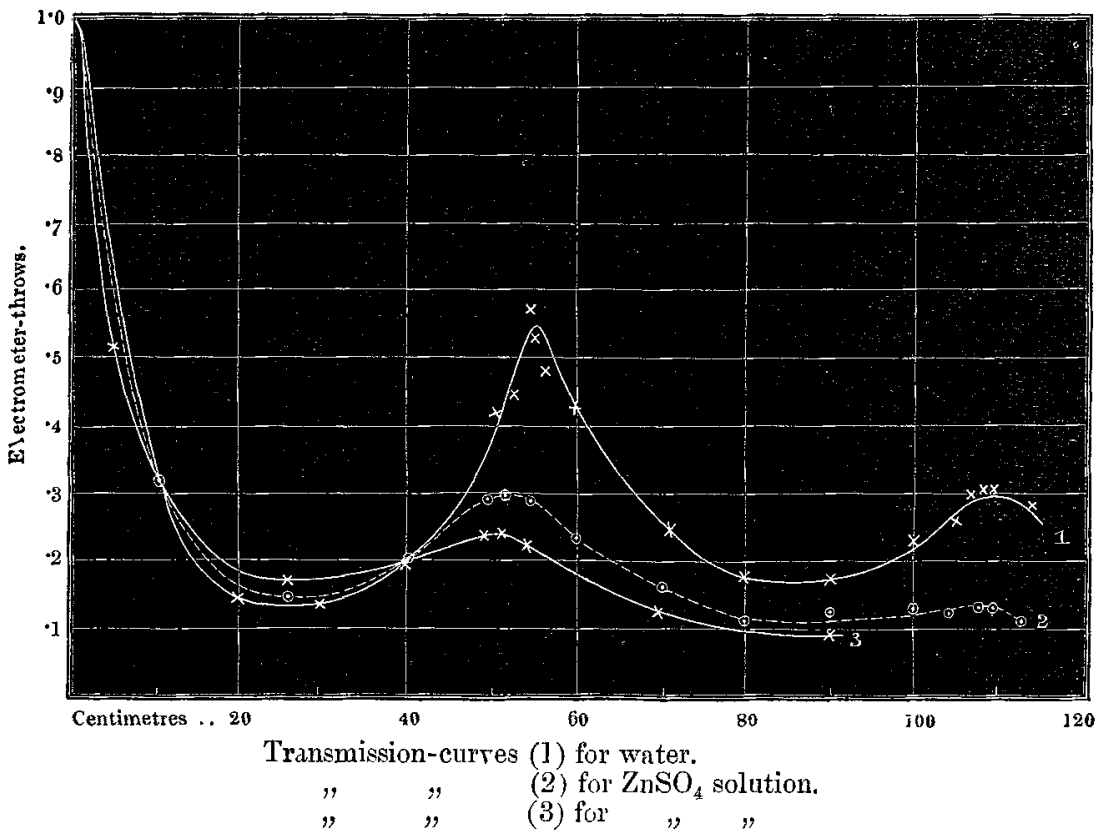


The complete results for distilled water are given in curve 1, fig. 3. It is seen that, at least for such a poor conductor as distilled water, any slight absorption is conpletely masked by the interference. As I mentioned in the introduction, the intensity of the transmitted ray does not uniformly decrease, but the transmission follows the same general law as for light with a thin plate: we are in fact dealing with a "thin plate"- a plate whose thickness is comparable with the wave-length of the radiation used. A minimum is transmitted by a plate a quarter wave-length thick, a maximum by a plate a half wave-length thick, and so on. The fact that the maxima in our curve (1. fig. 3) get successively lower is only in part due to the absorption : it is chiefly owing to the fact that we are here dealing, not with a steady wave-train like light, but with a rapidly damped wave-train. If the head of such a wave-train be only interfered with by the tail, it is not much affected: complete interference is quite impossible.

It was desirable to know how far the phenomenon would still be noticeable with electrolytes of higher conductivity. To test this question, a few drops of a strong solution of zine sulphate were added to the jarful (some 14 litres) of distilled water, and the relative intensities of the transmitted radiation determined as before. The results are given in curve 2, fig. 3. Curve 3 of the same figure was determined with a slightly stronger solution. In both curves the first maximum is very 'well marked. Both solutions w'ere, however, very dilute : their specific gravities with reference to water at the same temperature were approximately 1.00028 and 1.00038 , and their specific resistances 5100 and $4030 \mathrm{ohms} \times \mathrm{cm}$. respectively, as measured with the Kohlrausch bridge and telephone. To give an idea of the corresponding opacities I may add that radiation trarersing the first solution would be reduced to 0.41 of its initial intensity in a metre, or to 0.32 of its initial intensity in traversing a metre of the second solution.

With a view to making certain of the nature of the periodic effect, experiments were also carried out with liquids of a different specific inductive capaeity. Curve 1, fig. 4, was determined with 95 per cent. alcohol, and curve 2 with a mixture of three volumes of the same alcohol with one volume of water. In both cases the maxima are very well marked. 
Fig. 4.

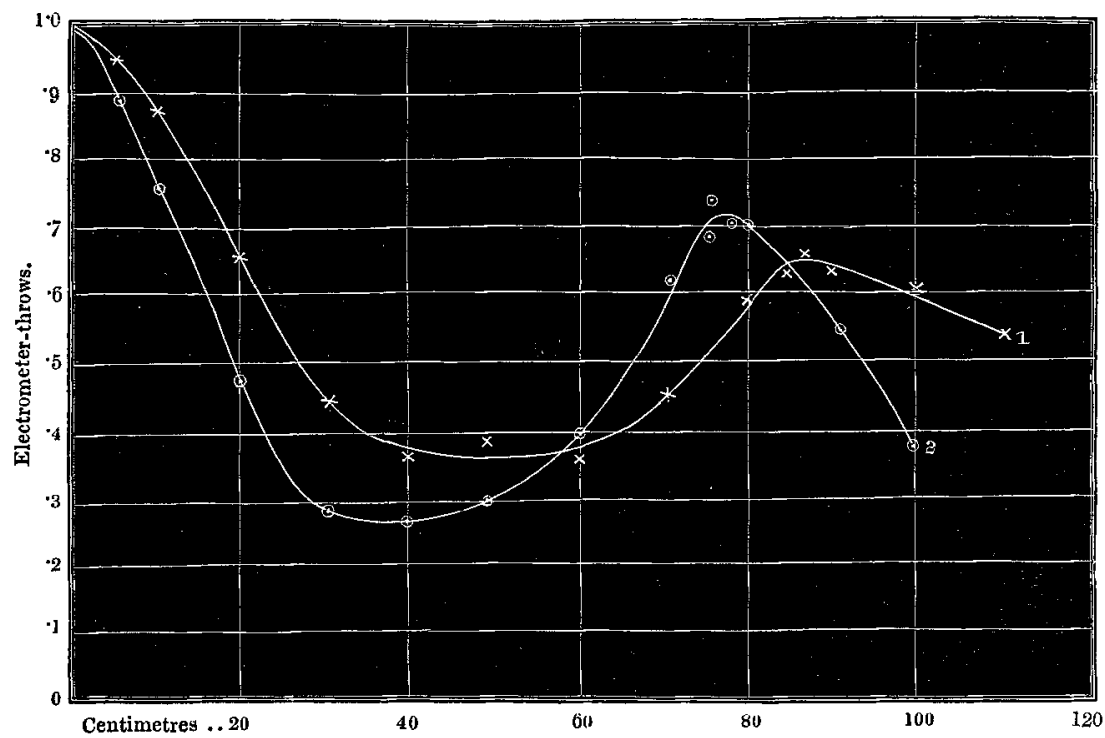

Transmission-curve (1) for 95 per cent. alcohol.

$" \quad \quad \quad$ (2) for $\left\{\begin{array}{l}3 \text { vols. } 95 \text { per cent. alcohol. } \\ 1 \text { vol. water. }\end{array}\right.$

\section{Numerical Results.}

For the very low conductivities with which we are here dealing, we may safely regard the values given by the maxima of the curves as the true wave-lengths, a correction for conductivity being quite negligible. As the wave-length in air is known to us, we may determine the coefficients of refraction $(n)$ and the dielectric constants $(k)$ of the various liquids used by the ordinary relation

$$
k=n^{2}=\left(\frac{\lambda_{a}}{\lambda_{s}}\right)^{2},
$$

where $\lambda_{a}, \lambda_{s}$ are the wave-lengths in air and the liquid respectively. These wave-lengths are given in the first column of Table II., and the corresponding values of the two constants in the second and third columns.

The experiments with zinc-sulphate solution show that an addition of salt which largely affects the conductivity of a 
TABLE II.

\begin{tabular}{|c|c|c|c|}
\hline & $\lambda \mathrm{cms}$ & $n$ & $k$ \\
\hline 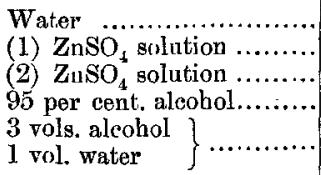 & $\begin{array}{l}108 \\
106 \\
104 \\
174 \\
154\end{array}$ & $\begin{array}{l}8 \cdot 33 \\
8 \cdot 49 \\
8 \cdot 65 \\
5 \cdot 17 \\
5 \cdot 84\end{array}$ & $\begin{array}{l}69 \cdot 5 \\
7 \cdot 2 \cdot 0 \\
74 \cdot 9 \\
26 \cdot 7 \\
34 \cdot 1\end{array}$ \\
\hline Air............. & 900 & - & - \\
\hline
\end{tabular}

solution only slightly increases its dielectric constant, thus confirming entirely the similar result published in 1892 by Cohn* (to whom we are also indebted for the first determination of the high constant of water $\dagger$ ).

For comparison with my figures I give in Table III. the dielectric constants of water and alcohol found by other investigators.

Table III.

\begin{tabular}{|c|c|c|c|}
\hline \multirow{2}{*}{ Method used. } & \multirow{2}{*}{ Authority. } & \multicolumn{2}{|c|}{ Dielectric Constants. } \\
\hline & & Water. & Alcohol. \\
\hline $\begin{array}{l}\text { Aiternated or } \\
\text { interrupted } \\
\text { currents. }\end{array}$ & $\begin{array}{l}\text { Heerwagen }{ }^{1} \text {. } \\
\text { Rosa }{ }^{2} \text {. } \\
\text { Rosa }{ }^{3} \text {. } \\
\text { Bouty }{ }^{4} \text {. }\end{array}$ & $\begin{array}{l}79 \cdot 56 \\
75 \cdot 70 \\
70 \\
\ldots\end{array}$ & $\begin{array}{c}257 \\
30 \cdot 9 \\
7.93\end{array}$ \\
\hline Ruhmkorff coil \{ & $\begin{array}{l}\text { Tereschin }{ }^{5} \text {. } \\
\text { Cohn \& Arons }{ }^{6} \text {. }\end{array}$ & $\begin{array}{l}83 \cdot 8 \\
76\end{array}$ & $\begin{array}{l}27^{*} \\
265^{*}\end{array}$ \\
\hline $\begin{array}{c}\text { Hertzian oscil- } \\
\text { lations. }\end{array}$ & $\begin{array}{l}\text { Ellinger }{ }^{7} \text {. } \\
\text { Cobn }^{8} \text {. } \\
\text { Stschegtiaeff }^{9} \text {. }\end{array}$ & $\begin{array}{l}81 \\
73 \cdot 5 \\
1 \cdot 75\end{array}$ & $\begin{array}{c}24 \\
1 \dddot{62}\end{array}$ \\
\hline
\end{tabular}

* 98 per cent.

+93 per cent.

1 Wied. Ann. xlviii. p. 35 (1893).

${ }^{3}$ Ibid. xxxiv, p. 344 (1892).

4 Jour, de Phys. $3^{\mathrm{e}}$ série, tome i. p. 445 (1892).

5 Wied. Ann. xxxvi. p. 792 (1889). ${ }^{6}$ Ibid. xxxiii. p. 13 (1888).

7 Ibid. xlvi. p. 513 (1892). ${ }^{8}$ Ibid. xlv, p. 370 (1892).

9 Phil. Mag. xxxiv. p. 388 (1892), or Journ. de Phys. June 1892.

* Wied. Ann. xlv. p. 370 (1892).

† Cohn \& Arons, ibid. xxxiii. p. 13 (1888). 
The figures given by Stschegtiaeff agree so nicely with Maxwell's law that one regrets being compelled to treat them as negligible; and the low value found for alcohol by Bouty is also in a minority and requires explanation. It should be noted that it was not determined directly, but deduced from the constants found for mixtures of small proportions of alcohol with other liquids.

My figures agree roughly with the high values given in the table, but are somewhat low. This is very likely to be ascribed to the fact that a certain portion of the wave always flows through the dielectric ontside the jar instead of going through the liquid. This portion interferes with the first in such a way as to shift the maximum of the curve forwards ; i.e. the whole arrangement behaves as if the wave had flowed through a layer of air and water mixed instead of through pure water.

The indefinite error arising from this cause might have been avoided in one way, namely, by making a certain length $A B$ (fig. 5) of the one wire into a tube surrounding the other, and using this tube $A B C D$ also for holding the liquids. The whole of the induction must then lie between the inside wire and the tube, and, consequently, the whole of the radiation must pass through the liquid.

This was in fact the arrangement that $I$ originally wished to use. It possesses, however, several serious disadvantages which finally led to its rejection in favour of the simple wires and glass jar. For one thing, such a condenser as the tube forms reflects of itself a considerable portion of the incident energy. Again, the variations in the position of the electrolyte surface, with reference to the top of the jar, would introduce fresh interference phenomena, as wavetrains are reflected equally well from the latter and the former (as in the apparatus used by Fig. 5.

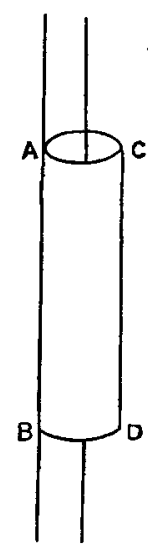
Mr. Barton*). The large surface of metal in contact with the liquid would cause additional inconvenience, as it would rapidly render distilled water or very dilute electrolytes impure.

In addition to the liquids $I$ have mentioned, salt and soda crystals were used with a view to try and determine their dielectric constants. No definite results were, however, 
obtained. With salt absolutely no effect could be observed, and soda gave only a slight drooping of the curve. This shows at all events that the constants of these substances approximate to normal values, and not to the very high figures found for water and alcohol.

\section{Note on the Theory.}

It was my original intention to calculate from the curves, not the dielectric constants, but the conductivities of the liquids. At first sight one might claim that this should be possible, but practically the matter is too complex to serve the purpose. On the assumption that the conductivity be very small, so that one may neglect the phase alteration on reflexion and the increase in the reflected fraction, the equation to the curve may be calculated by a procedure recently described by Mr. Barton*. I will only give the principal equations here, and refer to his paper for a more complete sketch of this method and discussion of fundamental pointst. We may write the equation of the incident wave-train in the form

where

$$
\begin{aligned}
& y=a e^{-\alpha t+\alpha_{1} x} \sin \left(\beta t-\beta_{1} x\right), . \quad . \quad . \quad . \\
& \alpha / \alpha_{1}=\beta / \beta_{1}=v_{1}, \quad . \quad . \quad . \quad . \quad \text {. }
\end{aligned}
$$

$v_{1}$ being the velocity of propagation in the ordinary portion of the long leads. Similarly, for the waves in the liquid,

$$
\alpha / \alpha_{2}=\beta / \beta_{2}=v_{2} \text {. . . . . . . }(2 a)
$$

For the primary damping $\gamma$, or time-rate of decrease of the amplitudes of oscillations in the primary, we have

where $\tau$ is the period.

$$
\gamma=\frac{2 \pi \alpha}{\beta}=\alpha \tau, \text {. . . . . . }
$$

Secondary damping, or damping out of the whole wavetrain, owing to the leads between which it travels not being perfect conductors, is legitimately neglected in this part of the theory as it is known to be small.

We assume the electrolyte to have a certain, though small,

* Proc. Roy. Soc. liv. p. 85 (1893), vide more particularly p. 90 . I am deeply indebted to Mr. Barton for full explanations of his theory prior to publication.

$\uparrow$ The case discussed by him is one of similar interference where there is no absorption in the medium. 
conductivity. Let the damping of wave-trains in their passage through it be given by a factor $p$, such that

$$
a_{2}=a_{1} e^{-p x}, \text {. . . . . . . }
$$

where $a_{1}$ is the initial amplitude, and $a_{2}$ the amplitude of the same wave after traversing a length $x$ of electrolyte. From Maxwell's theory it follows that

$$
p=2 \pi \mu \mathrm{CV}, \text {. . . . . . }
$$

where $\mathrm{V}=$ velocity of propagation in the electrolyte,

$\mathrm{C}=$ conductivity of the electrolyte.

$\mu=$ magnetic permeability of the electrolyte.

Taking the second surface of the electrolyte $\left(x_{2}\right.$, fig. 1$)$ as origin of coordinates, and carrying out the summation of successively emergent rays, we arrive, after sundry transformations, at the expression for the curve

where

$$
\frac{\mathrm{I}_{t}}{\mathrm{I}_{0}}=\frac{\left(1-b^{2}\right)^{2} e^{-2 p l}}{1-b^{4} e^{-4 p l}}\left\{1+\frac{2 Q}{\bar{\beta}}\right\}, . .
$$

$$
\mathrm{Q}=b^{2} e^{-2 p l-t_{2} \alpha}\left\{\frac{\alpha \sin t_{2} \beta+\beta \cos t_{2} \beta-\beta b^{2} e^{-2 p l-t_{2} \alpha}}{1-2 b^{2} e^{-2 p l-t_{2} x} \cos t_{2} \beta+b^{4} e^{-4 p l-2 t_{2} x}}\right\}, .
$$
and

$I_{0}=$ intensity transmitted when the absorbent laye. vanishes.

$\mathrm{I}_{t}=$ intensity transmitted for a thickness $l$ of the layer.

$b=$ fraction of the incident amplitude reflected at the first surface.

$$
t_{2}=\frac{2 l}{v_{2}}, \text { i.e. }=\text { the time taken by radiation to traverse }
$$
twice the thickness of the layer.

On putting the conductivity, or $p,=$ zero the above expression (6) reduces to that given by Mr. Barton for the case of no absorption.

On analysing (6) we see that the expression on the righthand side may be split into two terms : the first gives us a continuous drooping curve which may be considered as a centre line, on which the humps formed by the second, periodic, term are imposed. If we put the primary damping $\gamma=\infty$, interference is impossible, the periodic terms vanish, and we have

$$
\begin{aligned}
\frac{I_{t}}{I_{0}}= & \frac{\left(1-b^{2}\right)^{2} e^{-2 p l}}{1-b^{4} e^{-4 p l}}, \ldots . . . . . \\
& * \text { Maxwell, ii. [798]. }
\end{aligned}
$$


an equation which would give the form of the curve if no interference took place. It is not a simple logarithmic curve, inasmuch as we have to take account of multiple reflexions inside the absorbent layer. If we again put the conductivity $=0$, the centre line becomes a straight line parallel to the base instead of a drooping curve.

If we put in (7) $l=\infty, Q$ vanishes; $i . e$. as the length of the layer is increased the humps on the curve disappear. As I have already mentioned, this is due to the fact that the wave-train is a rapidly damped one so that interference is not perfect.

I have calculated a curve for the first zinc-sulphate solution by these equations; the theoretical and experimental curves are shown together in fig. 6 . The dielectric constant of the

Fig. 6.

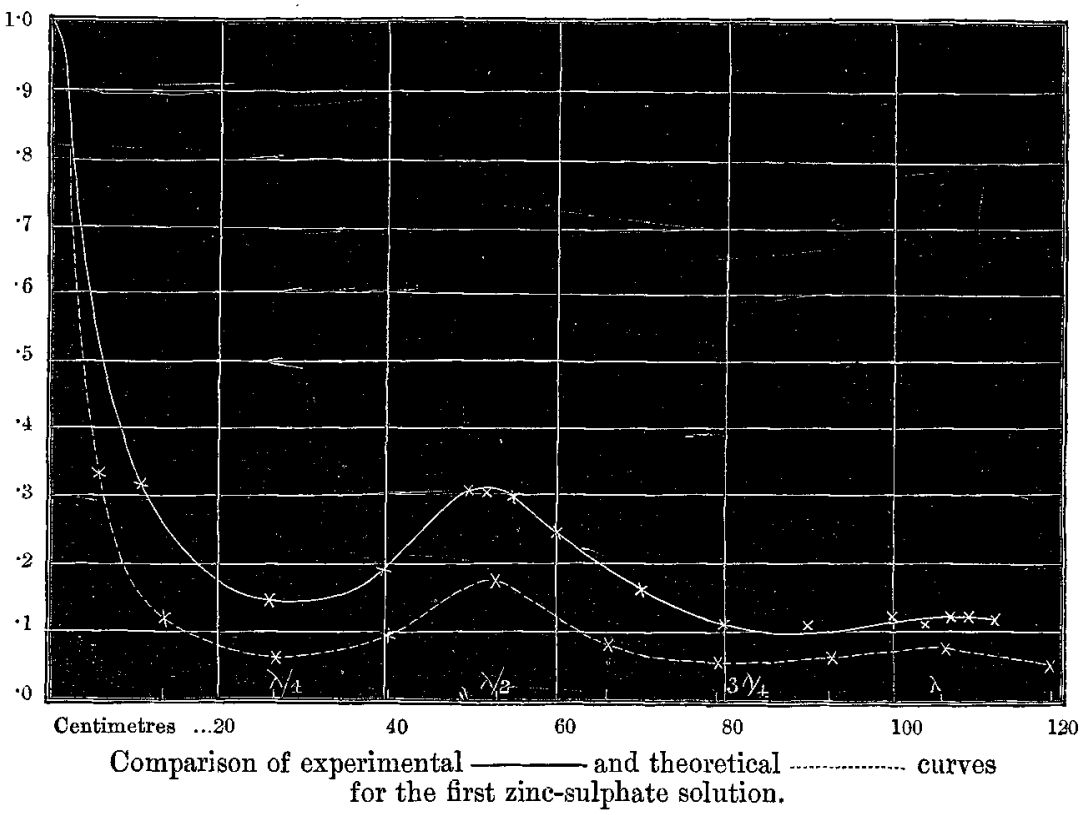

Data for Theoretical Curve.

Wave-length ........... $\lambda=106 \mathrm{cms}$.

By Kohlrausch bridge ....... $\sigma=5100 \mathrm{ohm}$. ems.

Assumed log. dec. .......... $\gamma=0.4$.

solution is known from the experimental curve; this gives us $b, v_{2}$, and $t_{2}$. The primary damping $\gamma$ we do not know, but an 


\section{Passage of Electric Wave-trains through Electrolyte.}

approximate value can be found by trial and error ;

$$
\gamma=0.4
$$

was found to be suitable. $\gamma$ furnishes us with $\alpha$ and $\beta$ from equations (2) and (3). The conductivity of the solution was measured with the Kohlrausch bridge (vide p. 537), and hence $p$ was known. We have, then, all the necessary data.

The two curves, calculated and experimental, disagree considerably; the experimental curve stands all along much higher than the other. Now this shift could not be caused by any variation in the conductivity: that only causes a quicker or slower downward trend of the curve. It could be approximately bronght about by an alteration in the reflected fraction, but by a decrease of it; i.e. if we took the conductivity into account in calculating the amplitude of the reflected ray, it would only make matters worse.

The fact of the matter is that we have all along neglected the multiple reflexions that occur in all parts of the circuit. The fraction reflected from the first surface of the electrolyte is not lost for good, but is turned back again when it reaches the secondary plates $\mathbf{B ~ B}^{\prime}$ (fig. 1), and is given another chance of getting through the liquid layer. Similar multiple reflexions occur not only between $\mathrm{B}$ and $x_{1}$ (fig. 1), but between $x_{2}$ and $\mathrm{D}$, and $\mathrm{B}$ and $\mathrm{D}$. The matter becomes too complex for calculation as we have a fresh unknown-the secondary damping-introduced. The fact that the experimental curve trends downwards towards the theoretical agrees with the above explanation : as the thickness of the absorbent layer is increased the waves are so weakened by their passage through it that multiple reflexions are of less account.

Though, as I have said, calculation is impossible, a comparison of the two curves in fig. 6 shows at least that no great alteration of conductivity has taken place.

\section{Conclusion.}

This investigation was carried out during the winter and summer semesters (1892-93) in the Physical Institute of the University at Bonn. It is with great pleasure that I take this opportunity of expressing my thanks to Prof. Hertz for his most useful advice and suggestions, and for the interest he showed in the work throughout. 